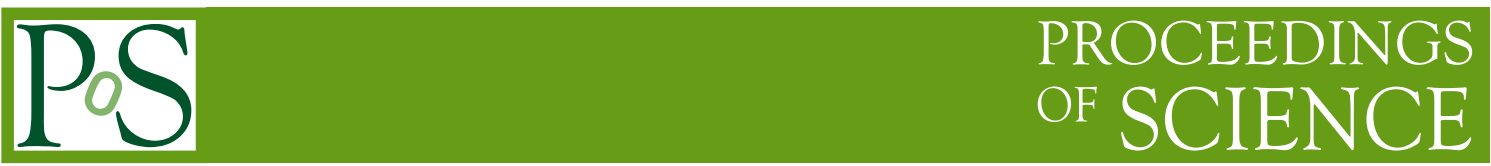

\title{
Performance of the CMS Silicon Tracker
}

\author{
Giacomo Sguazzoni*i \\ INFN Sezione di Firenze, Firenze, Italy \\ E-mail: giacomo.sguazzoni@cern.ch
}

The CMS Tracker is the largest Silicon detector ever built, for a total of $\sim 200$ square meters of silicon sensors, providing an average of 14 high-precision measurements per track. Track reconstruction is based on an iterative algorithm designed to optimize efficiency and estimate of track parameters. The alignment procedures and their performances are presented. Tracking and basic b-tagging observables are typically validated by using data-driven methods. Selected examples are discussed. The CMS Tracker is also capable of effective detection of photon conversions and nuclear interactions. Application examples of these features are given.

The 20th Anniversary International Workshop on Vertex Detectors - VERTEX 2011

June 19 - 24, 2011

Rust, Lake Neusiedl, Austria

\footnotetext{
${ }^{*}$ Speaker.

On behalf of the CMS Collaboration.
} 


\section{The CMS Tracker}

The Compact Muon Solenoid, CMS, is one of the two general-purpose experiments installed at the Large Hadron Collider (LHC) at CERN [1]. The core of the CMS detector is the superconducting solenoid, $6 \mathrm{~m}$ in diameter and $13 \mathrm{~m}$ long, that produces a magnetic field of $3.8 \mathrm{~T}$. The solenoid contains, from outside to inside, the brass-scintillator hadron calorimeter, the crystal electromagnetic calorimeter and the silicon tracking system for the reconstruction of charged particles trajectories. The gaseous detectors to identify and track the muons are placed outside the solenoid, embedded in the iron yoke.

The silicon tracking system, shown in Figure 1, is composed of a Pixel Silicon detector with three barrel layers at radii between $4.4 \mathrm{~cm}$ and $10.2 \mathrm{~cm}$ and two endcap disks at each end. Pixel sensors feature single pixel size of $100 \times 150 \mu \mathrm{m}^{2}$ for a total of $66 \mathrm{M}$ channels. The Silicon Strip Tracker covers the radial range between $20 \mathrm{~cm}$ and $110 \mathrm{~cm}$ around the $\mathrm{LHC}$ interaction point. The barrel region $(|z|<110 \mathrm{~cm})$ is split into a Tracker Inner Barrel (TIB), made of four detector layers, and a Tracker Outer Barrel (TOB), made of six detector layers. The TIB is complemented by three Tracker Inner Disks per side (TID). The forward and backward regions $(120 \mathrm{~cm}<|\mathrm{z}|<280 \mathrm{~cm})$ are covered by nine Tracker End-Cap (TEC) disks per side thus extending the overall acceptance to cover the region $|\eta|<2.5$. In some of the layers and in the innermost rings, special doublesided modules are able to provide accurate three-dimensional position measurement of the charged particle hits. The Silicon Strip Tracker is the world's largest silicon strip detector with a volume of approximately $23 \mathrm{~m}^{3}$, instrumented by about 15,000 modules with different strip pitches ranging from 80 to $180 \mu \mathrm{m}$, for a total of $198 \mathrm{~m}^{2}$ of Silicon active area and about 9.6 million channels with full optical analog readout [1][2]. The granularity was chosen to balance the need for a low occupancy, which is estimated to be a few percent at the largest expected LHC luminosity, and the requirement of minimising the power density and the amount of material.

The tracking detector features a transverse momentum resolution of about 1-2\% for muons of $P_{T} \sim 100 \mathrm{GeV}$, an impact parameter resolution of about $10-20 \mu \mathrm{m}$ for tracks with $P_{T} \sim 10-$ $20 \mathrm{GeV}$, a reconstruction efficiency of tracks within jets of about $0.85-0.90$ with a few percent fake

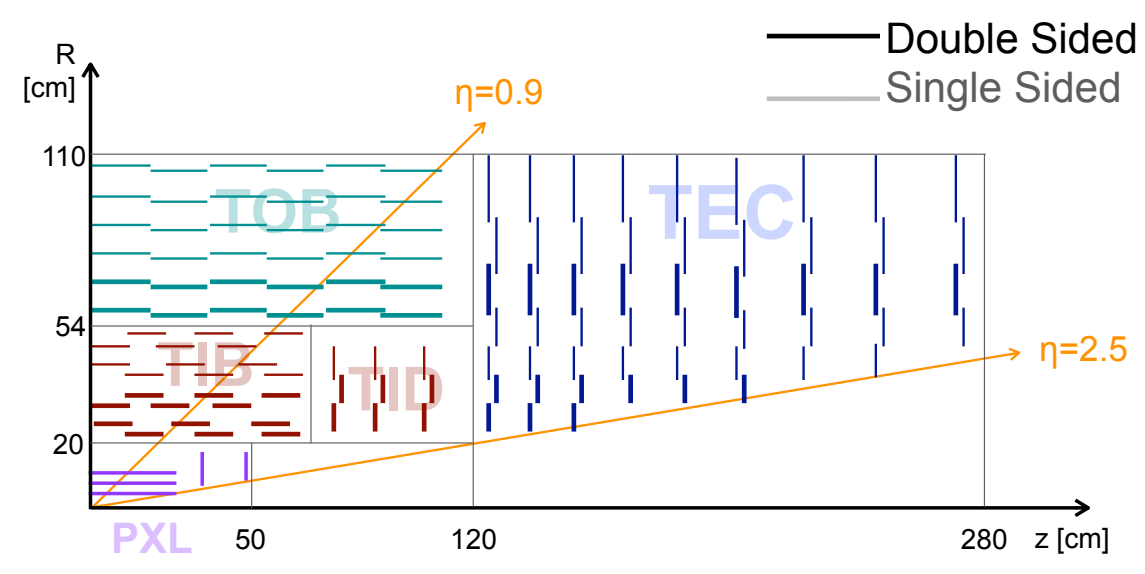

Figure 1: A simplified sketch of a quadrant of the $R z$ section of the CMS Tracker (bold lines represent double sided module assemblies). 


\begin{tabular}{cccccc} 
\#step & seed type & seed subdetectors & $P_{T}^{\min }[\mathrm{GeV} / c]$ & $d_{0} \mathrm{cut}$ & $z_{0} \mathrm{cut}$ \\
\hline 0 & triplet & pixel & 0.8 & $0.2 \mathrm{~cm}$ & $3.0 \sigma$ \\
1 & pair & pixel & 0.6 & $0.05 \mathrm{~cm}$ & $0.2 \mathrm{~cm}$ \\
2 & triplet & pixel & 0.075 & $0.2 \mathrm{~cm}$ & $3.3 \sigma$ \\
3 & triplet & pixel/TIB/TID/TEC & $0.25-0.35$ & $2.0 \mathrm{~cm}$ & $10.0 \mathrm{~cm}$ \\
4 & pair & TIB/TID/TEC & 0.5 & $2.0 \mathrm{~cm}$ & $12.0 \mathrm{~cm}$ \\
5 & pair & TOB/TEC & 0.6 & $2.0 \mathrm{~cm}$ & $30.0 \mathrm{~cm}$
\end{tabular}

Table 1: Relevant parameters of the six tracking iterative steps in CMS.

rate.

The operation of the CMS Pixel detector and of the CMS Silicon Tracker is described elsewhere [3] [4].

\section{Tracking at CMS}

The track reconstruction starts with the appropriate grouping of the hits in the innermost layers to build up seeds. The seed is an initial track estimate and consists of a pair or a triplet of hits, sufficient for a basic prediction of the trajectory parameters if the primary vertex is also used.

Starting from a given seed, pattern recognition using a Kalman Filter is performed to build inside-out trajectories. Then each identified track undergoes a procedure to reject possible outlier hits and is refitted, also using a Kalman Filter. Finally, a quality selection is performed.

The fitted track parameters are the charge over transverse momentum $q / P_{T}$, the pseudorapidity $\eta$, the azimuthal angle $\phi$ and the longitudinal and transverse impact parameters $d_{z}$ and $d_{x y}$ at the distance of closest approach to the primary vertex. The track parameters are then propagated through the magnetic field using the Runge-Kutta propagator during the pattern recognition and
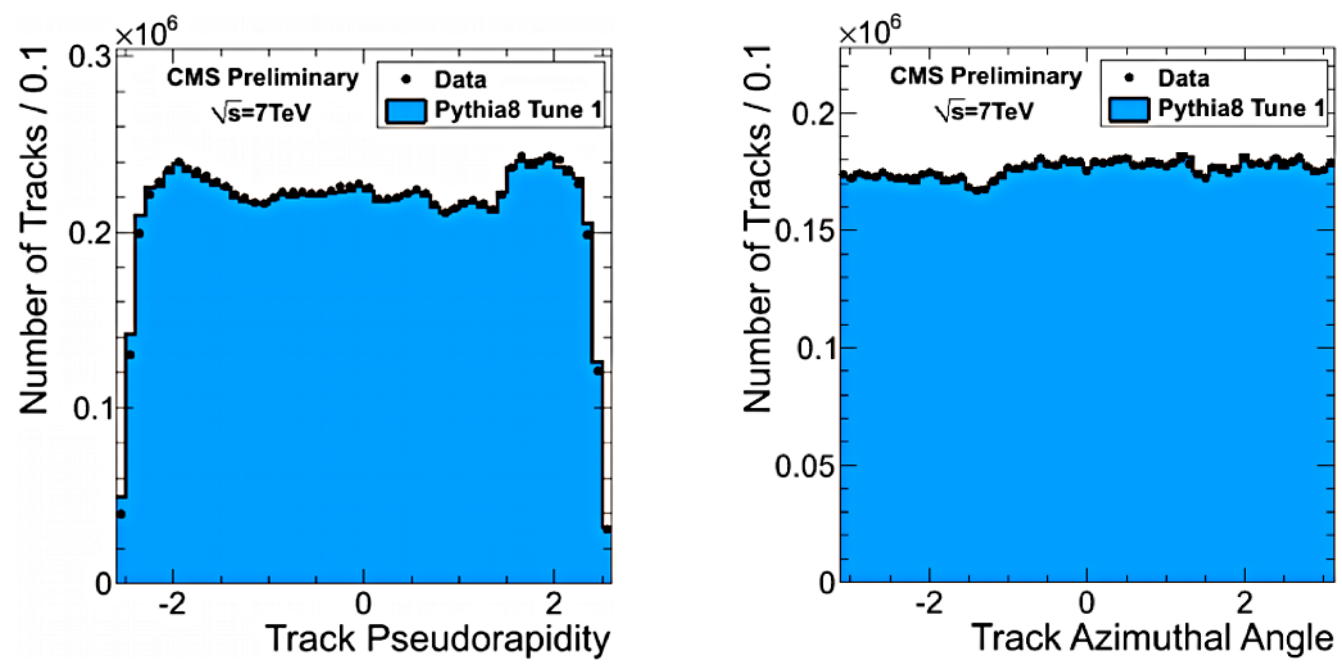

Figure 2: Number of reconstructed tracks in early LHC data (dots) and Monte Carlo simulation (histogram) as a function of $\eta$ (left panel) and $\phi$ (right panel). 
fitting phases. Reconstruction efficiency relies on six iterations (steps) of the tracking procedure; every step, except the first, works on the not-yet-associated hits surviving the previous step. Each step is optimized with respect to the seeding topology and to the final quality cuts. The relevant step configurations and parameters are reported in Table 1.

The number of reconstructed tracks as a function of $\eta$ and $\phi$ in 7 TeV pp data collected during the 2010 LHC run are shown in Fig. 2. The plots, made without any cut in $P_{T}$, show a remarkable agreement between data and Monte Carlo simulation. All detector non-uniformities and inefficiencies due to modules not read out are included in the simulation. A comprehensive account of the tracking performance can be found elsewhere [5] [6].

\section{Alignment}

The hit position resolution of the Silicon Tracker, ranging from about 10 to $30 \mu \mathrm{m}$, requires the knowledge of the position of the 16588 sensors with a comparable accuracy. Two track-based alignment algorithms are used in CMS for the determination of the Tracker sensor positions [7].

The Millipede-II algorithm [8] performs a global minimization of the $\chi^{2}$ that includes the track parameters and the sensor position parameters. To achieve this, the dependence on the track parameters is linearized and a custom track model is used. The alignment parameters are determined by solving a matrix equation with 105 elements.

The Hit and Impact Points (HIP) algorithm [9] performs a local minimization of the $\chi^{2}$ with respect to the position of each sensor; the parameters of the track used to compute the residuals are fixed and determined by excluding the sensor under study from the track fit. The correlations are taken into account by iterating the procedure several times and using the results of the previous iteration to re-reconstruct the tracks used in the $\chi^{2}$ computation.

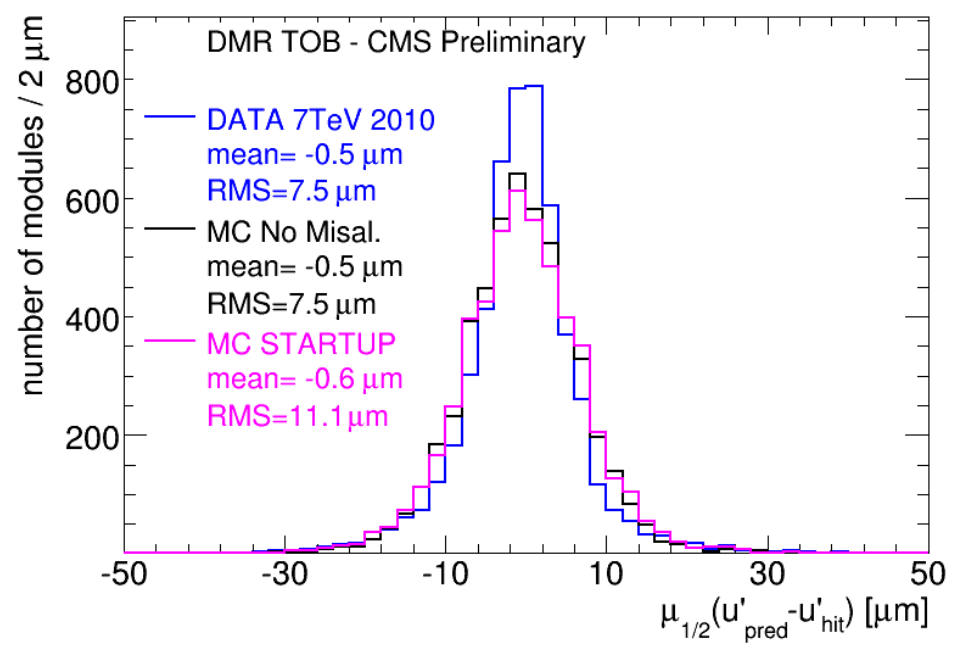

Figure 3: Distributions of the hit residual medians for sensors with more than 200 associated hits in TOB; post-alignment results from real data ("DATA") and simulation with realistic misalignment ("STARTUP”) are compared to raw simulation with ideal geometry ("MC No Misal.”). 


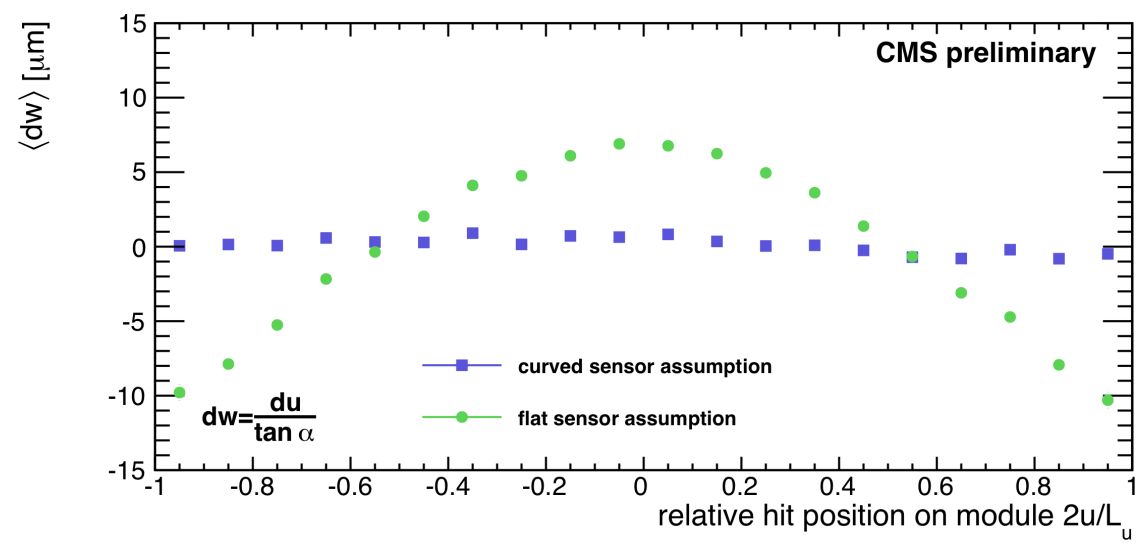

Figure 4: Average estimated deviation $\langle d w\rangle$ from the ideal sensor plane as a function of $2 u / L_{u}$; $u$ is the coordinate running in the ideal sensor plane, orthogonal with respect to the strips; $L_{u}$ is the sensor dimension in this direction. The quantity $d w$ is assumed to be $d u / \tan \alpha$ where $d u$ is the residual along $u$ and $\alpha$ the track incident angle. The plot refers to TIB modules, with and without correction for the bowed sensor hypothesis.

The two alignment algorithms are applied sequentially: first large scale subdetectors are aligned (Millipede), then the refinement at the level of the individual sensor is done using the HIP algorithm. The complementarity of the cosmic and collision track samples allows a good accuracy both in the barrel and in the endcaps of the Tracker to be achieved. Figure 3 shows the distribution of the median of the residuals (DMR) of each module with at least 200 hits associated to tracks, in the TOB subdetector. The DMR is shown after alignment using results from early LHC data ("DATA") and after alignment using results from a comparable amount of simulated data with a realistic misalignment scenario ("STARTUP"). These distributions are also compared to that from simulated data from a perfectly-aligned detector ("MC No Misal."). The DMR quantifies the statistical power of the alignment for a given sample of tracks used for the procedure. The similarity of the three distributions demonstrates that the alignment procedure is doing well and the performance is as good as can possibly be expected, given the amount of data used. The results for other subdetectors are similar [10].

The accuracy of the alignment procedure and the large number of modules in the CMS Tracker allow for subtle effects to be observed, such as bowing of the sensors [10]. By using tracks that are not orthogonal to the modules and assuming that the residual on the direction parallel to the sensor is only due to the local displacement along the normal direction, the sagitta of the sensor with respect to the ideal plane can be estimated. In Fig. 4 this sagitta for modules in the TIB subdetector is plotted before and after applying a correction for sensor bowing. Similarly, the aplanarity between the two sensors of TOB modules can be observed and appropriately taken into account. Since such corrections appear to be effective, they will soon become part of the standard alignment procedure.

\section{Tracking performances and validation}

The complexity of the CMS Tracker demands to reliably assess the track reconstruction per- 
formance not only by simulations but also by data-driven methods.

A standard method for measuring muon reconstruction efficiencies in data is the so-called tagand-probe technique [11]. A $J / \Psi$ is reconstructed requiring stringent quality cuts on one muon, referred to as the tag, and then the relative efficiency of two different selection criteria on the second muon, referred to as the probe, is determined. To measure tracking efficiency, probe muons are reconstructed by using only the muon detector system. The probe is matched to a track in the inner Tracker by requiring that $|\Delta \eta|<0.2$ and $\Delta \sqrt{\eta^{2}+\phi^{2}}<0.5$, which results in a track-muon matching efficiency of nearly $100 \%$ and a fake matching rate of approximately $10 \%$. The fraction of probes matching a track can be expressed as $\varepsilon=\varepsilon_{T} \varepsilon_{M}+\left(1-\varepsilon_{T} \varepsilon_{M}\right) \varepsilon_{F}$ where $\varepsilon_{T}$ is the true tracking efficiency, $\varepsilon_{M}$ is the matching efficiency, and $\varepsilon_{F}$ is the probability of random matches that can be also estimated from data measuring the off-resonance tracks that satisfy the matching criteria. The method, applied on $125 \mathrm{nb}^{-1}$ collision data, demonstrates that, within the kinematic range of muons used in the analysis $\left(P_{T}>1.5 \mathrm{GeV} / c\right)$, the tracking efficiency is $98.8 \pm 0.5 \%$, in agreement with the simulation.

The $J / \Psi$ mass and mass resolution are studied as a function of the muon kinematics and a calibration procedure can be applied to extract the momentum scale and measure the momentum resolution [12]. The procedure consists of a fit to resolution and momentum scale models that embed several possible systematic effects. Figure 5 (left panel) shows the mass as a function of $\eta$
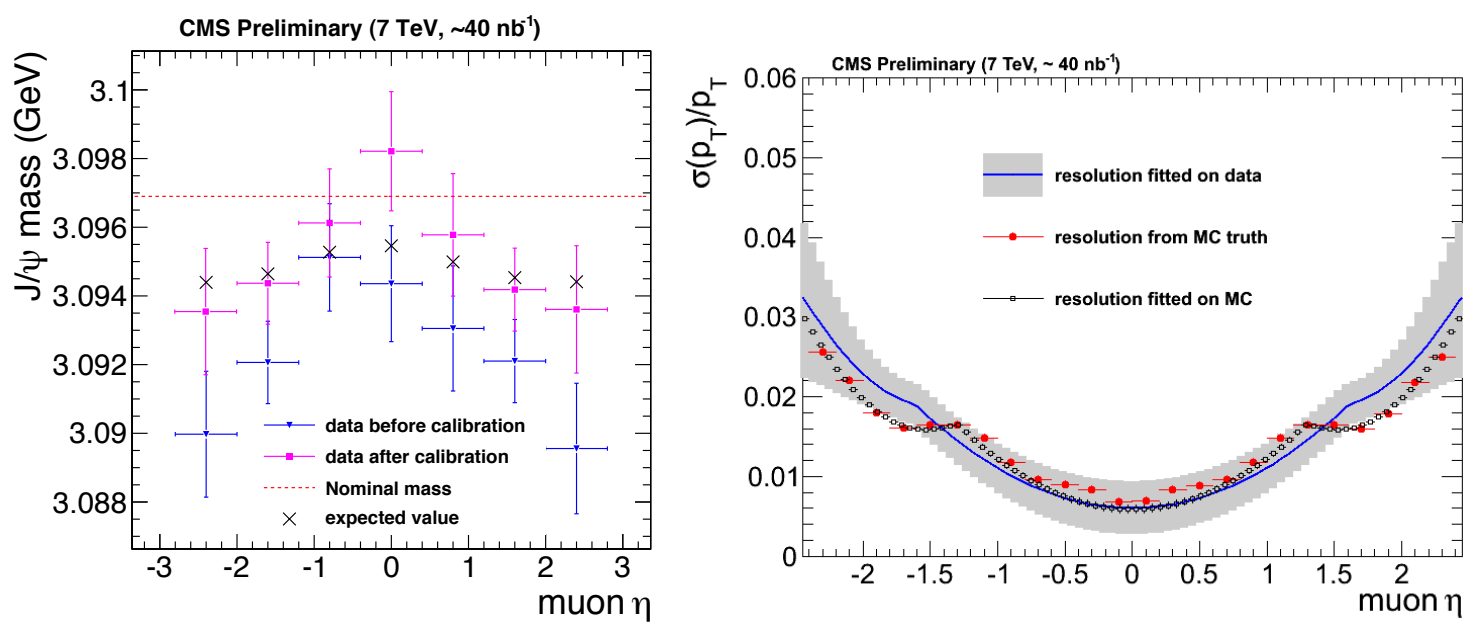

Figure 5: $J / \Psi$ mass as a function of $\eta$ before and after the scale corrections (left panel), compared with the expected values and the nominal mass value (dashed line). Resolution on transverse momentum (right panel) as measured with $\sim 40 \mathrm{nb}^{-1}$ of integrated luminosity (black line) compared to the resolution computed from Monte Carlo truth (red points) and extracted from the fit (black squares); the gray band in data represents the error on the fitted function for data computed from the errors on the track parameters.

before and after the scale corrections, compared with the expected values from a Crystal Ball fit to the lineshape model smeared with the resolution, for each $\eta$ value. The mass values after the corrections are much more in agreement with the expectation. The results for the transverse momentum resolution are shown in Fig. 5 (right panel). A discrepancy between data and simulation is visible in the high pseudorapidity region and especially in the transition region between barrel and 
endcaps. In that region the tracks intercept the detector cooling pipes and services, a considerable amount of passive material that could be not perfectly simulated.

Tracks reconstructed in an event are also used to determine the primary vertices by using an algorithm based on an Adaptive Kalman Filter. Primary vertex reconstruction is crucial for pile-up estimation and for b-tagging. A data-driven estimate of the of primary vertex reconstruction resolution and efficiency was implemented [6]. The tracks belonging to an already reconstructed vertex are randomly splitted into two subsamples, each used to attempt again a vertex reconstruction. The procedure can be used to evaluate the reconstruction efficiency as a function of the number of tracks (see Fig. 6) and, if the procedure is successful for both subsamples, the distance between the two vertices allows the position resolution to be estimated, as shown in Fig. 6 (right panel) for the $x$ coordinate. Results for the $y$ and $z$ coordinates are comparable.

The primary vertex reconstruction can be also used to derive the luminous region or beam spot. An alternative method for the beam spot determination exploits the fact that a track with azimuthal angle $\phi$ acquires an apparent transverse impact parameter $d_{x y}$ with respect to the nominal interaction point $(0,0)$ given by $d_{x y}=X \sin \phi-Y \cos \phi$ (here the dependence along the $z$ coordinate has been neglected for the sake of simplicity). $X$ and $Y$ are the coordinates of the actual interaction point that can be extracted by fitting the $d_{x y}$ vs. $\phi$ dependence. Similarly it can be shown that the correlation $<d_{x y}^{1} d_{x y}^{2}>$ between the impact parameters of two tracks can be expressed as a function of $\cos \left(\phi_{1} \pm \phi_{2}\right)$ with the resolutions on $X$ and $Y$ as parameters, again easily obtainable by a fit. This approach, not requiring any computationally demanding algorithm, is fast and can be used also online. An example of beam position and beam spot size measured with this method, as a function of time during a fill, is shown in Fig. 7 in comparison with the same quantities measured with a pixel-based vertexing algorithm. Among other features, the artificial displacement of beams in order to maximise instantaneous luminosity (luminosity scan), and the degradation of the beam quality with time can be seen in those plots.
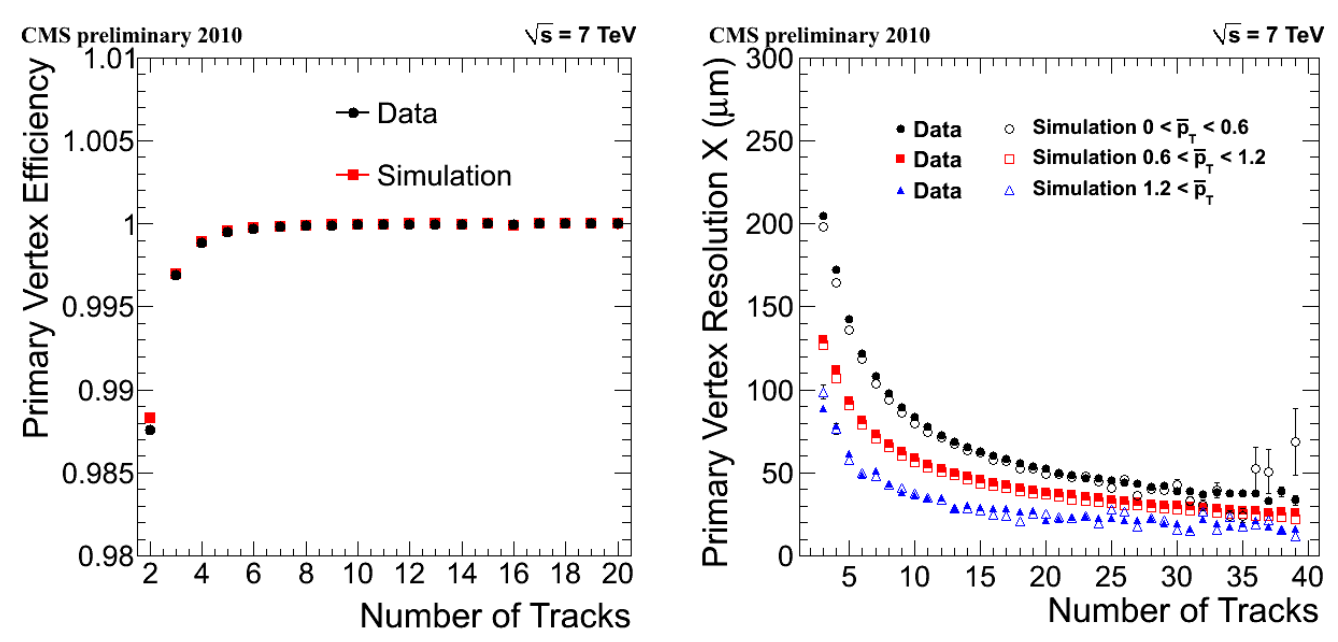

Figure 6: Primary vertex efficiency (left panel) and resolution in $x$ for different average transverse momentum (right panel) as a function of the number of tracks. 


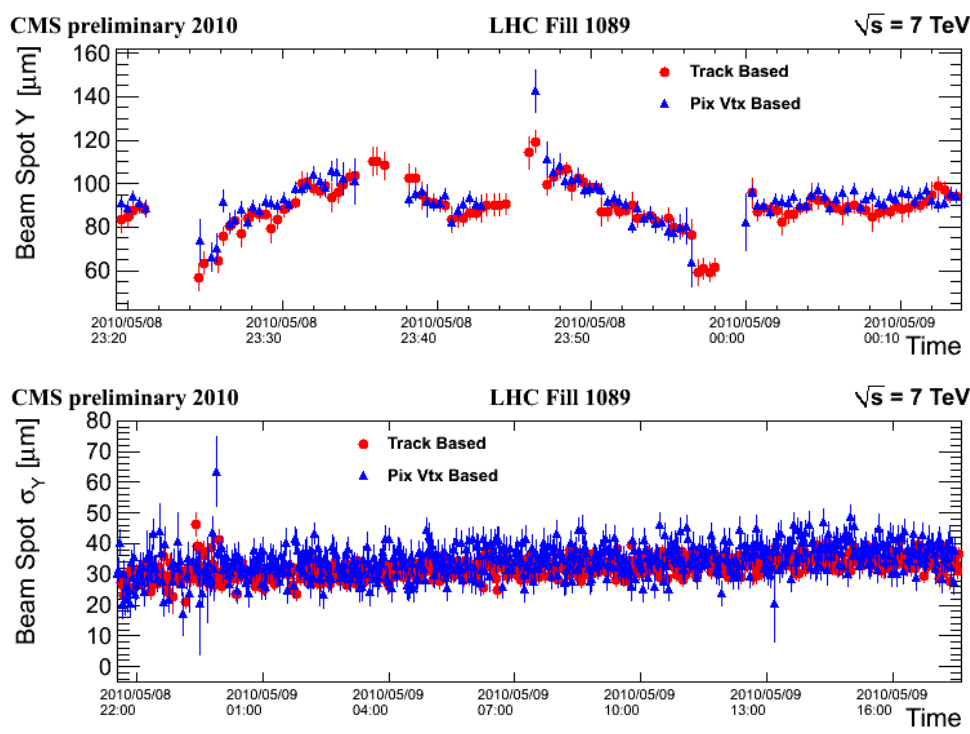

Figure 7: Fitted $y_{0}$ position of the beam line as a function of time during an LHC fill where a luminosity scan was performed (upper panel); fitted beam width along $y$ of the beam line as a function of time during one LHC fill. Blue dots and red dots represent result from the pixel-based vertexing method and from the track based method, respectively.

\section{B-tagging primitives}

The track-based primitives needed to construct b-tagging estimators can be validated using data-driven methods. The impact parameter (IP) resolution can be extracted by removing one track from a reconstructed primary vertex and studying the distribution of the impact parameter of that track with respect to the vertex refitted without that track [6]. The width of the distribution is given by the convolution of the IP resolution with the primary vertex resolution, that is also measurable from data as explained in Sect. 4. The contribution of non-prompt tracks is negligible.

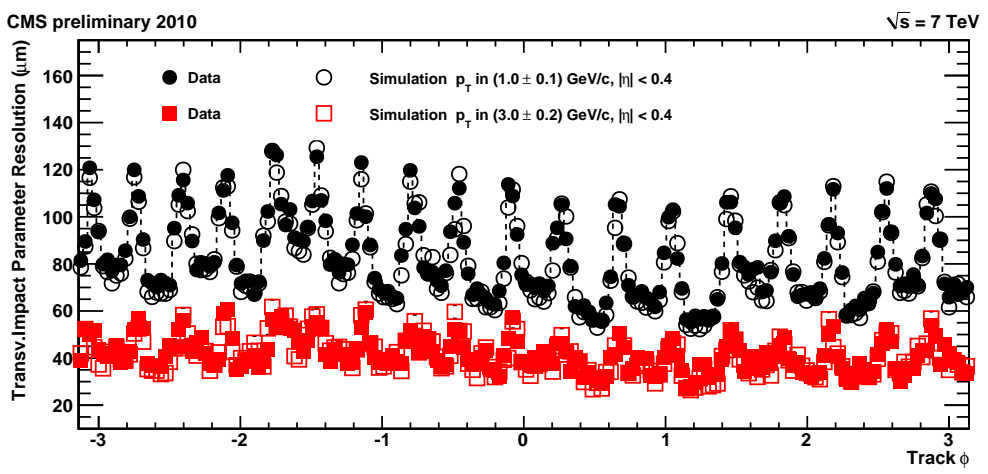

Figure 8: Measured resolution of the track transverse impact parameter as a function of the track $\phi$ for transverse momenta in $1.0 \pm 0.1 \mathrm{GeV} / c$ (circles) and in $3.0 \pm 0.2 \mathrm{GeV} / c$ (squares) range. Filled and open symbols correspond to results from data and simulation, respectively. 


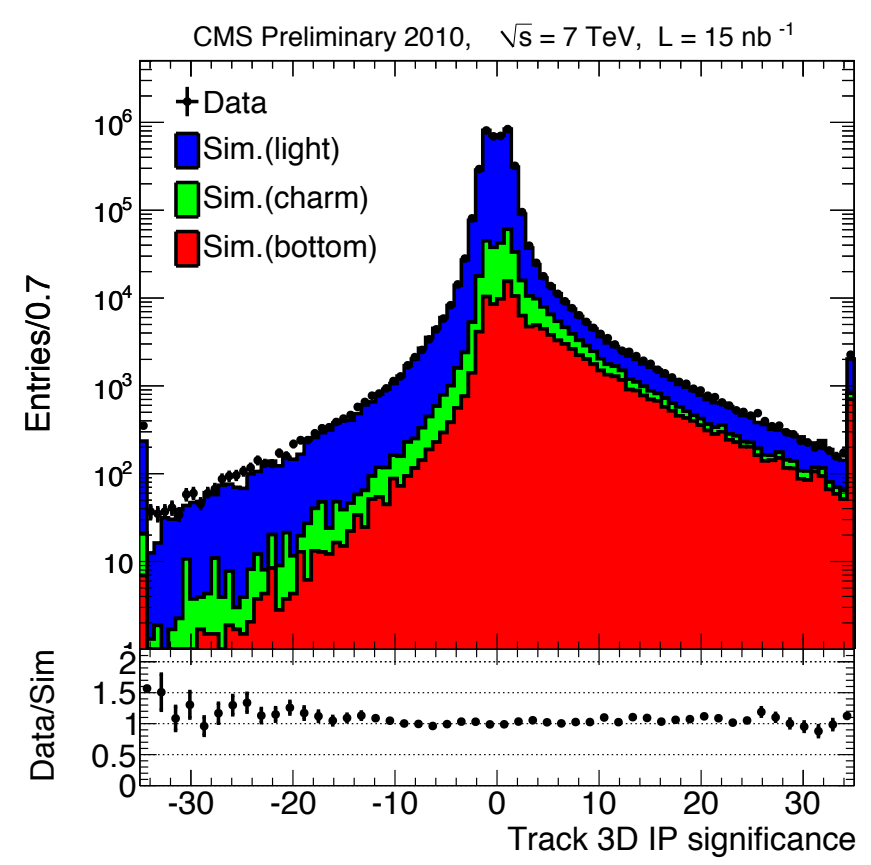

Figure 9: Three-dimensional impact parameter significance for data (dots) and simulated contribution from light quarks, charm and bottom in the blue, green and red stacked histograms, respectively.

The resolution estimated with this method is between 50 and $20 \mu \mathrm{m}$ for the transverse impact parameter and between 70 and $40 \mu \mathrm{m}$ for the longitudinal impact parameter $\left(|\eta|<0.4\right.$ and $P_{T}>$ $2 \mathrm{GeV} / c)$.

Figure 8 shows a particularly interesting plot illustrating the effect of the pixel detector material on the IP resolution. The resolution degrades noticeably in regions where tracks encounter the pixel detector cooling pipes, corresponding to the peaks in the plot.

In Fig. 9 the distribution of the three-dimensional impact parameter significance, the main component for an inclusive b-tagging estimator, is shown; in Fig. 10 the distribution of the threedimensional flight distance significance, important for any exclusive b-tagging measurement, is shown. The agreement between data points and Monte Carlo simulation is remarkable [13].

\section{Other CMS Tracker highlights}

The CMS Tracker has a fully analog readout and can therefore measure the track specific ionization loss, a powerful observable for particle identification, as discussed in [4] [14].

The highly granular CMS Tracker allows the reconstruction of vertices associated with both photon conversions and nuclear interactions. They are caused by the interactions of photons and hadrons, respectively, with the detector material [15]. In this respect they are a very useful handle to probe the detector material as shown in Fig. 11 (left panel). Photon conversion reconstruction is also crucial for analysis of final states where a soft photon is expected; this is the case in the reconstruction of the radiative decay of $\chi_{c}$ states into $J / \Psi$ such as $\chi_{c}^{1} \rightarrow J / \Psi \gamma$ and $\chi_{c}^{2} \rightarrow J / \Psi \gamma[16]$. 


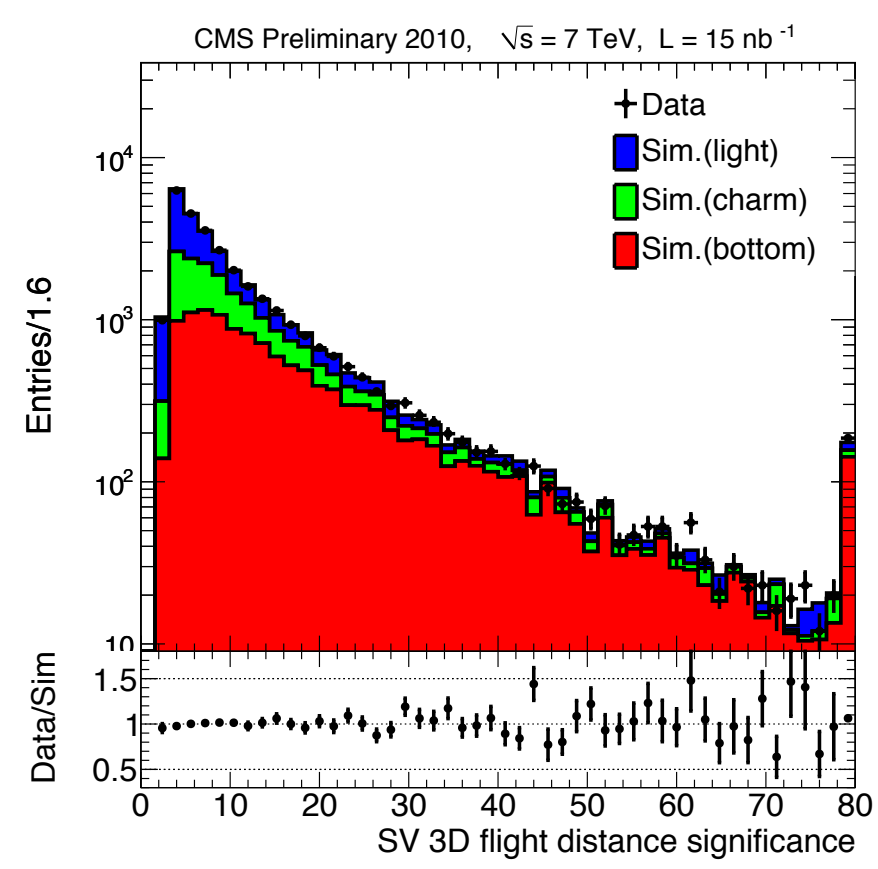

Figure 10: Three-dimensional flight distance significance for data (dots) and simulated contribution from light quarks, charm and bottom in the blue, green and red stacked histograms, respectively.

The excellent momentum resolution, achievable when the photon converted in the Tracker material is reconstructed, allows the two $\chi_{c}$ differing in mass of only $\sim 50 \mathrm{MeV}$ to be clearly separated, as shown in Fig. 11 (right panel).

\section{Conclusion}

The LHC collision data collected with CMS between 2009 and 2011 have demonstrated that the CMS Silicon Tracker performance, established directly from data, is outstanding and well understood. The CMS Silicon Tracker is able to provide the reliable reconstruction of tracks, vertices, and b-tagging primitives needed for the CMS physics analyses. Other features of the CMS Silicon Tracker, such as the measurement of $d E / d x$ or the ability to effectively reconstruct photon conversions and nuclear interactions, are also crucial for many physics results.

\section{References}

[1] R. Adolphi et al. [CMS Collaboration], The CMS experiment at the CERN LHC, JINST 3 (2008) S08004.

[2] CMS Collaboration, The Tracker System Project Technical Design Report, CERN-LHCC/98-6 (1998); CMS Collaboration, Addendum to the CMS Tracker TDR, CERN-LHCC/2000-16 (2000).

[3] A. Starodumov, Operations of the CMS Pixel detector, these proceedings.

[4] L. Demaria, Operations of the CMS Strip detector, these proceedings. 

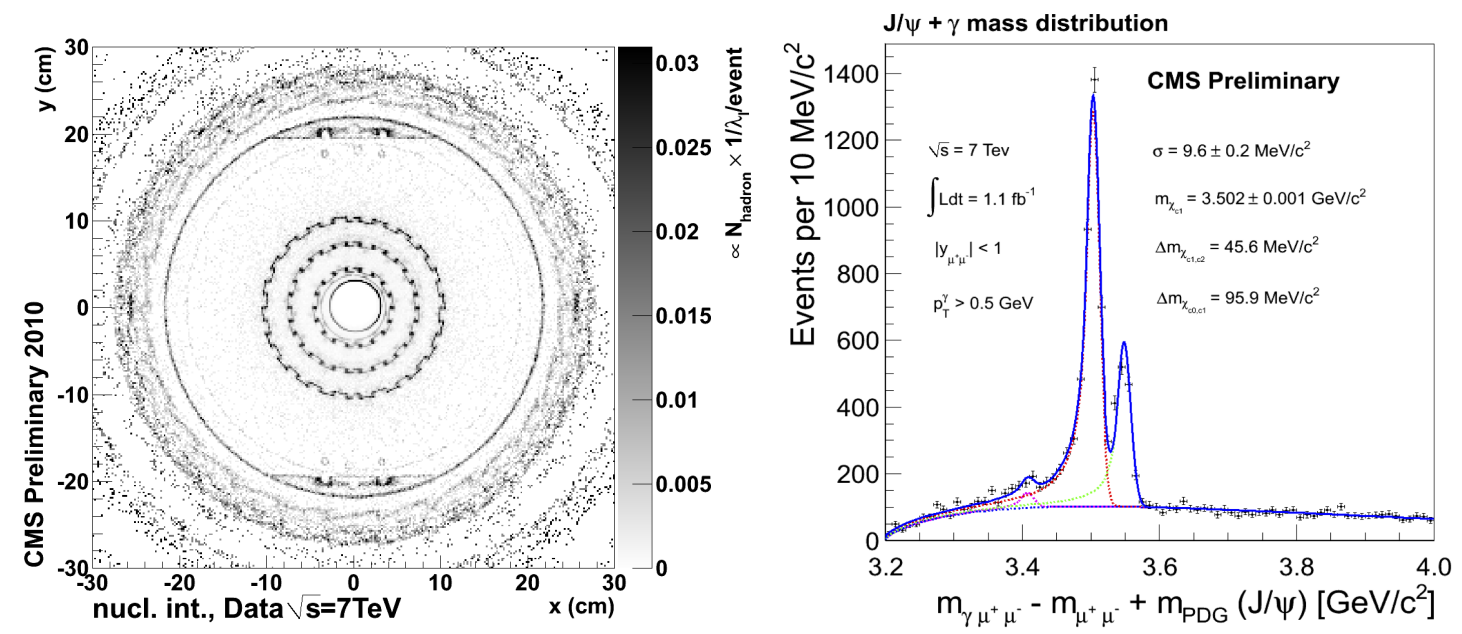

Figure 11: A $x y$ Tracker material map of the pixel barrel region obtained by counting the nuclear interactions (left panel); the mass peaks of the $\chi_{c}^{1}$ and $\chi_{c}^{2}$ decaying radiatively into $J / \Psi \gamma$, with the photon recosctructed via its conversion in the Tracker material (right panel); also the $\chi_{c}^{0}$ peak is visible.

[5] CMS Collaboration, Tracking and Vertexing Results from First Collisions, CMS-PAS-TRK-10-001 (2010).

[6] CMS Collaboration, Tracking and Primary Vertex Results in First $7 \mathrm{TeV}$ Collisions, CMS-PAS-TRK-10-005 (2010).

[7] S. Chatrchyan et al. [CMS Collaboration], Alignment of the CMS Silicon Tracker during Commissioning with Cosmic Rays, JINST 5 (2010) T03009.

[8] V. Blobel, Software alignment for tracking detectors, Nucl. Instrum. Meth. A566 (2006) 5-13.

[9] D. N. Brown, A. V. Gritsan, Z. J. Guo et al., Local alignment of the BABAR silicon vertex tracker, Nucl. Instrum. Meth. A603 (2009) 467-484.

[10] CMS Collaboration, CMS Tracker Detector Performance Public Results, http://twiki.cern.ch/twiki/bin/view/CMSPublic/DPGResultsTRK.

[11] CMS Collaboration, Measurement of Tracking Efficiency, CMS-PAS-TRK-10-002 (2010).

[12] CMS Collaboration, Measurement of Momentum Scale and Resolution using Low-mass Resonances and Cosmic Ray Muons, CMS-PAS-TRK-10-004 (2010).

[13] CMS Collaboration, Commissioning of b-jet identification with pp collisions at $\sqrt{s}=7 \mathrm{TeV}$, CMS-PAS-BTV-10-001 (2010).

[14] A. Giammanco, Particle Identification with Energy Loss in the CMS Silicon Strip Tracker, CMS Note 2008/005 (2008).

[15] CMS Collaboration, Studies of Tracker Material, CMS-PAS-TRK-10-003 (2010).

[16] CMS Collaboration, CMS B-Physics and Quarkonia Public Results, http://twiki.cern.ch/twiki/bin/view/CMSPublic/PhysicsResultsBPH. 